injected into the subcutaneous tissue in the flank. This is followed in a few days by the development of an abscess filled with aseptic pus, and usually accompanied by slight pyrexia. About the seventh day the abscess is incised and the contents evacuated. The whole procedure is conducted under strict aseptic precautions; the contents of the abscess should be sterile. In young or weakly patients the injection may be reduced to half a cubic centimetre of turpentine, given alone, or with the addition of an equal quantity of olive oil.

The advocates of this addition to the "therapeutic arsenal of ophthalmology" consider it a very valuable resource in iridochoroiditis and sympathetic ophthalmitis. "It does not always result in a definite cure, but in every instance it has led to an arrest in the progress of the malady."

With this emphatic statement before us the desirability of an extended trial of this method in the treatment of destructive forms of uveitis is obvious.

\title{
Miners' Nystagmus
}

The incidence of miners' nystagmus, the commonest of all industrial diseases, has shown a marked increase from 1908, the year after the disease was scheduled, when 460 men received compensation, to 1913, the last year of published statistics, when 4,551 men were disabled. Llewellyn, in a recent lecture, estimated that, including incipient cases and men who had cọmmuted, 6,000 men have been disabled yearly since 1913 , and that the cost to the country has been $£ 1,000,000$ a year.

The opinion, expressed by the Oxford Ophthalmological Congress of 1912, that deficient illumination is the chief factor in the disease is now held by most observers, with the notable exception of Dransart. The holing or undercutting of the coal has not the importance alleged by Snell, who thought that the disease was almost entirely confined to holers; in fact, 50 per cent of Llewellyn's 1,400 consecutive cases had done no holing. Errors of : refraction, accidents, and ill-health appear to be predisposing factors.

Two distinct varieties of the disease are described; the latent, in which symptoms are absent, and the manifest in which some incapacity exists. The chief symptoms are loss of sight, especially at night time, headache, dread of light, giddiness on stooping, and movement of the eyes. It is important to realize that the nervous system is profoundly affected in the disease. The characteristic physical sign of nystagmus is often masked by clonic spasm of the eyelids.

Miners' nystagmus is not readily cured, and the proportion of 
men who return permanently to work underground does not exceed twenty-five per cent. Many men reach a state of "equilibrium" and continue to work for years after the onset of the disease. Remedy lies in prevention, and it has been suggested that the illumination of the candle pit, where nystagmus is rare, should be taken as a standard for the safety lamp pit.

The question of improvements in the miners' safety lamp is arousing great interest at the present time, and a Home Office Committee is dealing with the subject.

\section{ABSTRACTS}

\section{I-ORBITAL ANAPLEROSIS}

Lagrange (Bordeaux).-Orbital anaplerosis. orbitaire.) Arch. d'Ophtal., March-April, 1919.

(De l'anaplérose

Under the term anaplerosis (derived from the Greek $\dot{a} \nu a \pi \lambda \eta \rho^{\prime} \omega$, to fill up), Lagrange describes $\mathrm{a}^{\prime}$ new procedure designed, like many other operations introduced during the last forty years, to provide a substitute for the enucleated eyeball, and to form a satisfactory stump on which an artificial eye may rest, and from which the latter may derive movement. The author reviews briefly the methods which have hitherto been employed; the pseudo-plastic devices, such as Mules's operation, the heteroplastic grafts, e.g., rabbit's eyes, and more recently homoplastic grafts of skin and fat, and of cartilage. None of these methods has proved really satisfactory; the best aesthetic results have been obtained in cases in which abscission of the anterior parts of the eyeball has been performed and this procedure, the writer recognizes, is suitable for a very limited number. The operation now described by Lagrange seems to the reviewer to be based on sound principles and to give greater promise of success in filling up the cavity of the orbit than any others with which he is acquainted. Unfortunately, the procedure is one which no written description, unaided by diagrams, will portray adequately and intelligibly. Those who are interested should study the original article.

The operation is suitable for cases of immediate enucleation, or for those in which the eyeball has been removed previously. The technique, though lengthy, is simple, and is given in detail by the writer and illustrated by numerous helpful diagrams. It consists essentially in obtaining a pedicled flap of connective tissue and fat from the region adjoining the external angle of the orbit. This flap is exposed by a T-shaped incision through the skin. In the space 